\title{
Alignment Strategy for the ATLAS Tracker
}

\author{
Pawel Brückman de Renstrom ${ }^{1}$ \\ University of Oxford, \\ DWB, Keble Road, Oxford OX1 3RH, UK \\ and \\ Institute of Nuclear Physics PAN, ul. Rdzikowskiego 152, Kraków 31-342, PL \\ EE-mail: p.bruckman1@physics.ox.ac.uk
}

\begin{abstract}
ATLAS is a general purpose spectrometer in preparation to take data on the Large Hadron Collider at CERN. It will start its operation in autumn 2007. Charged particle tracking is realised by the Inner Detector. ID consists of two silicon subsystems: Pixel Detector and Semiconductor Tracker complemented by straw proportional gas chambers. In order to exploit the excellent intrinsic resolution of the precision tracking devices a high accuracy alignment is required. In this report the strategy to align silicon detectors of the ATLAS ID will be reviewed together with the current status of preparation.
\end{abstract}

\section{Introduction}

The silicon tracking system of the ATLAS Inner Detector (ID) $(1 ; 2)$ is composed of two subsystems: the Pixel Detector and the the SemiConductor Tracker (SCT). Altogether it consists of 5832 individual silicon modules which amounts to 34992 degrees of freedom (DoF $)^{2}$. These are arranged in three Pixel and four SCT cylinders in the barrel part and three Pixel and 9 SCT disks in each of the end-caps. $50 \times 400 \mu \mathrm{m}$ pixels provide a $2 \mathrm{D}$ readout with $14 \times 115 \mu \mathrm{m}$ resolution respectively ${ }^{3}$. SCT modules consist of two single-sided back-to-back strip detectors with a relative $40 \mathrm{mrad}$ stereo angle.

\footnotetext{
$\overline{1}$ On behalf of the ATLAS Inner Detector alignment community

2 Each module, assumed to be rigid, has three translational and three rotational DoF's.

3 Based on single pixel binary readout. This can be further improved (3)
}

Barrel SCT modules have parallel strips with $80 \mu \mathrm{m}$ pitch while end-cap ones have a fan-out structure. In the barrel resolution in the direction perpendicular to strips is $23 \mu \mathrm{m}$ per side while the stereo angle provides $580 \mu \mathrm{m}$ resolution along strips. More details about the Pixel and the SCT systems can be found elsewhere $(3 ; 4)$.

Alignment corrections are defined in the local reference frames of the modules with local $X$ along the sensitive direction ${ }^{4}$ and local $Y$ being the orthogonal in-plane direction.

\section{The Alignment Strategy}

The Transition Radiation Tracker (TRT) straw tubes are arranged into 96 barrel modules and 28 end-cap disks. These make the basis of alignment parameters. Being a drift device, TRT also needs

\footnotetext{
${ }^{4}$ Parallel to the short pixel side and across SCT strips.
} 
T0 calibration at a single straw level and RT ${ }^{5}$ calibration with some coarse granularity. Consequently, TRT alignment and calibration represents different challenges to the ones associated with the silicon system. The current strategy assumes an intrinsic alignment of the silicon tracker followed by TRT alignment to the tracks extrapolated from the silicon. An alternative approach integrating alignment of both silicon and TRT in single algorithm is now undergoing initial validation ${ }^{6}$.

The overall strategy for the alignment of the silicon tracking system relies on track-based offline alignment algorithms complemented by the Frequency Scanning Interferometry (FSI) system in the SCT. The two have complementary roles as argued in section 4 . The initial alignment is provided by the mechanical and optical surveys of the asbuilt geometry.

\section{Track Based Alignment Algorithms}

Three independent algorithms for track based alignment of the ID silicon detectors have been developed alongside. All three are based on reconstructed track-to-hit residuals which carry information about track fit quality, ergo quality of the alignment. All described methods have been implemented in the Athena (5) framework and make part of the official ATLAS software.

Track based alignment inherently suffers from so called "weak modes" which correspond to detector deformations which do not (significantly) compromise $\chi^{2}$ of track fits. Such deformations often lead to biases on reconstructed track parameters. Some of these can be eliminated by introducing a common vertex constraint, constraints on track parameters coming from external tracking systems or direct constraints on the alignment parameters. Cosmic events, representing off-axis continuous helices across the whole detector volume, also provide a powerful handle against some weak modes. Track based algorithms include implementations of the above mentioned functionalities.

\footnotetext{
5 Relationship betveen the drift time and the distance from the anode wire.

6 TRT is believed to help constraining track curvature, hence to eliminate notorious sagitta distortions.
}

\subsection{Real Data Challenges}

Track based algorithms were applied to two real experimental setups. In summer 2004 the Combined Test Beam (CTB) collected first ever real data from all ATLAS subsystems combined (6). The ID silicon geometry consisted of six Pixel modules and eight SCT ones arranged in a tower. SCT modules were exactly perpendicular to the beam line, while Pixel modules were arranged with a tilt angle of around $20 \mathrm{deg}$. CTB benefited from abundant event statistics at different beam momenta ${ }^{7}$ while a very small setup and the layout creating ill-defined modes (collimated beam through a narrow tower of modules) were of clear disadvantage. Data were taken with and without magnetic field. In June 2006 integrated SCT and TRT barrel detectors recorded over 400,000 cosmic events in the ATLAS surface building (SR1). SCT took data with 468 out of 2112 barrel modules in the two opposite wedges of the detector extending over its full length. Pixels were not present and there was no magnetic field to reconstruct cosmic ray momenta.

\subsection{Robust Alignment}

The robust alignment approach is an iterative method to align any kind of silicon detector with overlapping modules. In each iteration alignment corrections are calculated out of measurements of mean residuals $\bar{R}_{i}$ and mean $\phi$ and $z$ overlap residuals $\overline{O R_{i, \phi / z}} \cdot i$ stands for either $X$ or $Y$ labelling residuals along the local axes of the module as defined in section 1 . The method aims at correcting two to three DoF's only, i.e. two translations in the plane of the module and if possible systematic radial translations. The three different residual and overlap residual measurements are combined according to the formula:

$$
\begin{aligned}
& a_{j}=-\sum_{j=1}^{3} \frac{s_{j}}{\left(\delta s_{j}\right)^{2}} / \sum_{j=1}^{3} \frac{1}{\left(\delta s_{j}\right)^{2}} \\
& s_{1}=\bar{R} ; s_{2}=\sum \overline{O R_{\phi}} ; s_{3}=\sum \overline{O R_{z}} .
\end{aligned}
$$

Alignment corrections (1) relate module position to its adjacent neighbours via $\phi$ and $z$ overlaps. The

$72-180 \mathrm{GeV} / \mathrm{c}$ with $O\left(10^{5}\right)$ tracks/module/energy 

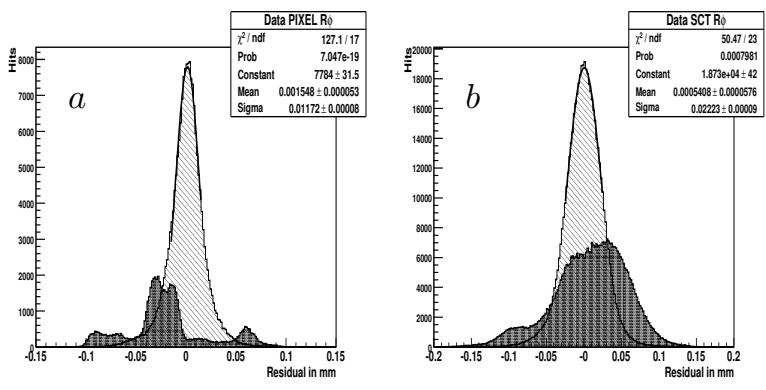

Fig. 1. Local $X$ residual distributions in CTB Pixel (a) and SCT $(b)$ detectors. Dark-shaded histograms are the initial geometry while the light-shaded ones after the Robust alignment has converged.

method heavily relies on iteration. More details can be found in (7).

The method was first validated on the CTB data. It provided fast and stable results. Figure 1 shows the improvement to the local $X$ residuals in Pixels and SCT due to the alignment. Convergence was always reached within 15 iterations.

Robust method has also been applied to the SR1 cosmic data. The analysis is ongoing but the preliminary results prove validity of the algorithm.

\subsection{Global $\chi^{2}$ Alignment}

The Global $\chi^{2}$ algorithm is based on the minimisation of the $\chi^{2}$ defined as:

$\chi^{2}=\sum_{\text {tracks }} r^{T} V^{-1} r$

with respect to the alignment parameters. $r$ is the vector of hit residuals to the fitted track and $V$ is its covariance matrix. The residuals depend on the track parameters $(\pi)$ as well as on the subset of alignment parameters related to the intersected module $(a)$. The generic solution for alignment corrections reads:

$\delta a=-\left(\sum_{\text {tracks }} \frac{d r^{T}}{d a} V^{-1} \frac{d r}{d a}\right) \sum_{\text {tracks }}^{-1} \frac{d r^{T}}{d a} V^{-1} r$

with $\frac{d r}{d a}=\frac{\partial r}{\partial a}+\frac{\partial r}{\partial \pi} \frac{d \pi}{d a}$

$\frac{d \pi}{d a}$ can be obtained by differentiating similar expression for a single track fit to the reconstructed hits in the detector. More details about the method can be found elsewhere (8). The method has a big advantage of properly treating all correlations between residuals arising from common track parameters and Coulomb scattering. There is a price to pay. The solution 3 requires inverting a symmetric matrix of size $\mathrm{N}$ where $\mathrm{N}$ is number of DoF's of the problem. In case of ATLAS ID it represents an important numerical challenge. To make things worse, the matrix is inherently singular which requires more careful treatment ${ }^{8}$.

Validation of the Global $\chi^{2}$ algorithm has been done on real data from CTB and SR1 cosmic runs as well as on fully simulated ATLAS data. On the CTB data the method converged within four iterations. Initial broad residual distributions transformed into clean Gaussians with widths of $10.7 \mu \mathrm{m}$ and $19.1 \mu \mathrm{m}$ for the Pixel modules and SCT respectively. These were consistent with the perfectly aligned simulation.

The approach was also used to align SR1 cosmic setup. Around 250,000 events were used in this analysis. Because initial misalignments were small the convergence was nearly instantaneous. Second iteration already delivered the final quality alignment. Results are summarised in figure 2 . Correction pulls are given in the diagonal base, i.e. the base of orthogonal modes of the detector deformation ${ }^{9}$ and are sorted from left to right by increasing eigenvalue. Structure in the right part of plot $a$ shows misalignment modes which the available data is actually sensitive to. Plot $c$ demonstrates that after second iteration alignment fully converged. After alignment the fitted width of the residuals agreed with the one from perfectly aligned simulation within one micron. The Monte Carlo sample was generated with the actual cosmic muon spectrum cut-off at $200 \mathrm{MeV}$. In real data we had some yet lower energy particles. These contributed to the far tails of the residual distribution but did not affect the width of the peak region.

The algorithm has also been tested on Monte Carlo data simulated through the complete ID geometry. So far tests consisted of checking properties of results obtained on perfectly aligned de-

\footnotetext{
8 E.g. diagonalisation. Other options include preconditioning or sequential solving with an arbitrary cut-off. Feasibility of the earlier was studied and documented elsewhere (9). 9 These are obtained by means of diagonalisation.
} 

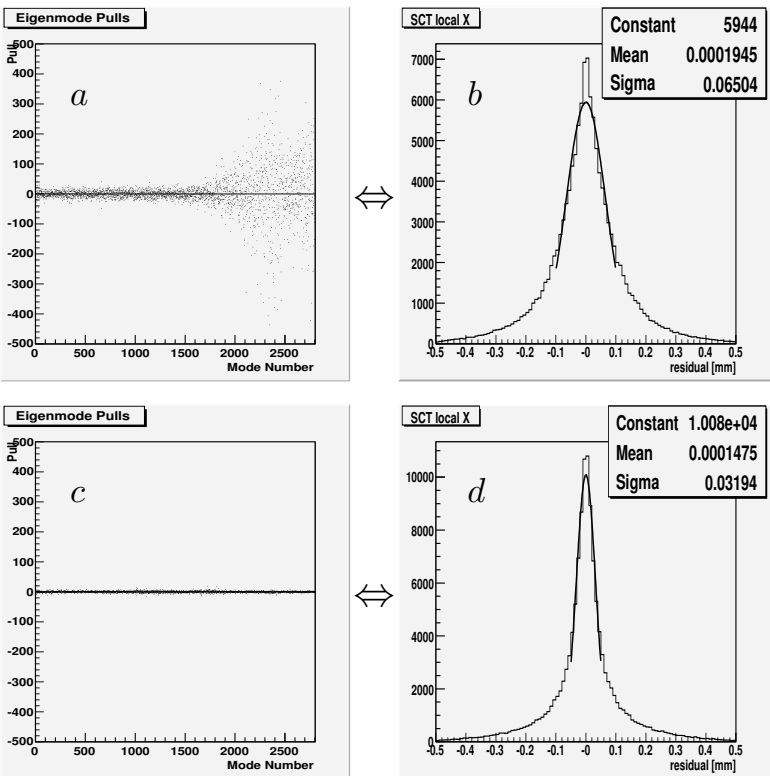

Fig. 2. Performance of the Global $\chi^{2}$ algorithm on the SR1 cosmic setup (468 SCT modules). Upper plots show correction pulls for the 2808 eigenmodes $(a)$ and corresponding local $X$ residual distribution $(b)$ as obtained from the initial geometry. Lower plots $(c$ and $d$ ) show analogous distributions on realigned geometry (after two iterations).

tector. Dedicated events containing on average 10 muons with $2 \mathrm{GeV} / \mathrm{c}<p_{T}<50 \mathrm{GeV} / \mathrm{c}$ were used. First, the full barrel part of the Pixel and the SCT detector (21408 DoF's) was realigned with a limited statistics of 640,000 tracks. The matrix was diagonalised using pdsyevd routine of the ScaLAPACK package on a AMD Opteron parallel cluster (9). Reconstructed pulls of the alignment corrections exhibited Gaussian distribution of unit width, as expected. A more recent test benefited from an ample 7,500,000 tracks simulated into the cone of $|\eta|<1.0$. The corresponding subset of Pixel and SCT detectors consisted of 2172 modules (13032 DoF's). With this high statistics the typical error on the absolute position of the module was of the order of $10 \mu \mathrm{m}$. We seem to have observed an over-statistical fluctuation in one of the low modes corresponding to $\phi$ rotation of the Pixel detector relative to SCT. This could sign a residual systematic effect at some stage of the upstream reconstruction. Fortunately, this kind of deformation can be efficiently constrained using a common vertex requirement.

\subsection{Local $\chi^{2}$ Alignment}

The Local $\chi^{2}$ algorithm is based on the same ansatz (3) but the full derivative (4) is reduced to just the partial derivative with respect to $a$ and full covariance matrix $V$ is replaced by its diagonal only. As a result the system of equations breaks down to $6 \times 6$ blocks which are straightforward to solve. The solution is now given by simply:

$\delta a=-\left(\sum_{\text {tracks }} \frac{\partial r_{i}^{T}}{\partial a} \frac{1}{\sigma_{i}^{2}} \frac{\partial r_{i}}{\partial a}\right)_{\text {tracks }}^{-1} \frac{1}{\sigma_{i}^{2}} \frac{\partial r_{i}^{T}}{\partial a} r_{i}$

This approximation is justified as long as tracking uncertainty is smaller than measurement uncertainty which usually is a valid assumption. Because all correlations via common track parameters as well as Coulomb scattering are ignored this method is inherently iterative in order to make up for the reduced information.

Local $\chi^{2}$ method proved its principle providing good performance on both CTB and SR1 cosmic data. Speed of convergence lies in between the Robust and the Global $\chi^{2}$ algorithms. For the CTB alignment all DoF's per module except for the translation along the beam line were used. Alignment quality after reaching convergence was consistent with prediction from the perfect simulation. On SR1 cosmic data the algorithm performed the complete alignment procedure involving all six DoF's per module. Figure 3 shows the convergence of the algorithm on real SR1 cosmic data. Full convergence was reached after $\sim 10$ iterations. A similar exercise was performed on simulation misaligned by as much as the specified assembly tolerances (4). Two main observations were made: The convergence pattern was similar and resulted in comparable track fit quality. The misalignments assumed for the simulation were substantially larger than the ones found in the real system. The latter indicates excellent precision of the entire SCT assembly.

The Local $\chi^{2}$ algorithm was also tried on the full ATLAS geometry using simulated events. The tests using simulation of perfectly aligned geometry confirmed good behaviour of the correction pull distributions and expected convergence pattern. 

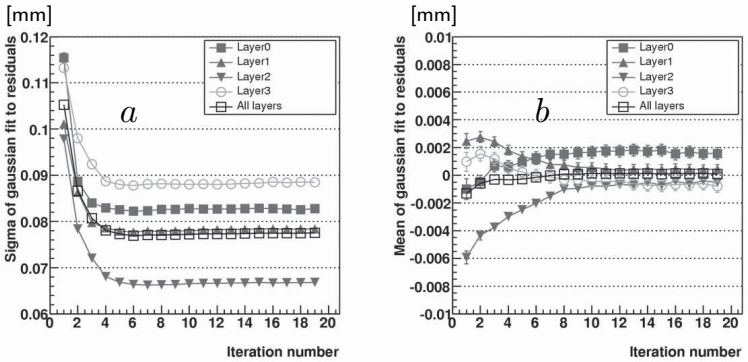

Fig. 3. Performance of the Local $\chi^{2}$ algorithm on the SR1 cosmic setup (468 SCT modules). Plots show the widths (a) and the means (b) of the Gaussian fit to the local $X$ residual distributions in the four SCT layers as a function of iteration number.

\section{Frequency Scanning Interferometry}

FSI is a novel stability monitoring system based on simultaneous measurement of multiple grid line interferometer lengths (10). The system consists of 842 grid lines arranged into a geodetic grid inside the SCT volume. It also contains a tunable laser and the reference interferometer of precisely known length. Length measurement is based on observing fringes in both the measured and the reference interferometers while the the tunable laser scans the frequency. Ratio of the observed phase change is equal to the ratio of interferometer lengths. Node positions can subsequently be reconstructed with accuracy better than $10 \mu \mathrm{m}$ in 3D (11).

FSI cannot provide absolute measurements of SCT module positions but delivers quasi real-time (timescale $\sim 10 \mathrm{~min}$ ) movement field for low spacial frequency eigenmodes which are very difficult to detect with the track based algorithms. Track alignment, in turn, provides complementary information about high spacial frequency eigenmodes on much longer timescales $(24 \mathrm{~h}+)$. The FSI system will allow for corrections to be made to short term motions of the support structures prior to the offline track alignment.

\section{Summary and Outlook}

ID of ATLAS adopted a variety of techniques in order to assure optimal alignment of its precision tracking devices. Three independent track based alignment algorithms are being developed to align Pixel+SCT tracker and a novel Frequency Scan- ning Interferometry system is integrated into the SCT part. All track based algorithms have provided convincing proof of principle. Preliminary burn-in of the algorithms was provided by the 2004 CTB. Currently we have started aligning the actual detector. Over 400k cosmic events were collected by integrated SCT and TRT detectors in June 2006. Alignment analyses confirmed assembly precision outclassed the specified mechanical tolerances. Large simulation datasets with misaligned geometries of the entire ID are being prepared now. First alignment results using these data are due early 2007. TRT and SCT barrel parts are already installed inside the ATLAS spectrometer. Pixel detector will be installed in spring 2007. FSI is getting ready to monitor $\mathrm{SCT}$ distortions due to this process. This will be followed by collecting cosmic events in the pit and ultimately by LHC accelerator events due late 2007.

\section{References}

[1] ATLAS Detector and Physics Performance TDR Vol. 1, CERN/LHCC/99-14.

[2] ATLAS ID TDR, CERN/LHCC/97-16\&17.

[3] M. Cristinziani, these proceedings.

[4] J. Carter, these proceedings.

[5] ATLAS Computing TDR-017, CERNLHCC-2005-022.

[6] B. Di Girolamo, M. Gallas, T. Koffas, ATCTT-IN-0001.

[7] D. Hindson, DPhil Thesis, Oxford university, 2004; F.Heinemann, ID Alignment Workshop, Ringberg, 2006.

[8] P. Brückman de Renstrom \& S. Haywood, PhyStat05 Proceedings, IC Press, L. Lyons \& M. K. Ünel (eds), 2006.

[9] M. Karagöz Ünel, P. Brückman de Renstrom, K. Bernadet, A.Hicheur, CHEP06 proceedings, India, 2005.

[10] P. Coe et al., MST 1511 2175-87, 2004; S. Gibson et al., OLE 437 815-31, 2005.

[11] S. Gibson, DPhil Thesis, Oxford University, 2004. 